\title{
Prenatal screening for cystic fibrosis: psychological effects on carriers and their
}

\section{partners}

\author{
M E Mennie, M E Compton, A Gilfillan, W A Liston, I Pullen, D A Whyte, \\ D J H Brock
}

\begin{abstract}
This study aimed to assess the psychological impact of screening for cystic fibrosis (CF) carrier status in a population of pregnant women. A cohort of 1798 women, who accepted the offer of testing before 18 weeks of pregnancy, filled in a self administered questionnaire seeking information on their perceived risk of carrier status and their emotional response, as well as a general health questionnaire (GHQ). Sixty-four women identified as $C F$ carriers had partners who received a negative test result. This group and their partners were assessed, together with selected controls, on four further occasions: (1) on receiving the carrier's positive test result; (2) on receiving the partner's negative test result; (3) six weeks later; (4) six weeks after delivery. The instruments used were the GHQ and the Symptom Rating Test (SRT).
\end{abstract}

When compared to control subjects, carriers showed a significant increase in generalised psychological disturbance which could be attributed specifically to symptoms of anxiety and depression during the period (average four days) that they awaited their partner's test result. On receiving a partner's negative test result, the carriers returned to control levels and maintained this equilibrium. Although there was no significant difference in generalised psychological disturbance between partners and their selected controls, partners did become significantly more anxious and manifested feelings of inadequacy while awaiting their own test result. Both male partners and male control subjects were more likely to become anxious if their partner was distressed.

(f Med Genet 1993;30:543-8)

Population carrier screening for cystic fibrosis (CF) has become possible since the identification of the cystic fibrosis gene ${ }^{1}$ and the discovery that a relatively small number of mutations account for the majority of CF chromosomes in the UK population. ${ }^{23}$ One objective of carrier screening is to provide information to individual persons or couples so that they can make reproductive plans for the future. Another objective is to allow pregnant women to avoid the birth of a child with CF.

There are a number of possible approaches to CF carrier screening and The Cystic Fibrosis Trust has funded three trials to assess the feasibility of delivering screening by alternative routes. ${ }^{45}$ In Edinburgh a prenatal approach has been adopted. ${ }^{5}$ The principal steps in this trial are: (1) to offer pregnant women, attending the antenatal booking clinics of a major maternity hospital, CF carrier screening by way of a mouthwash sample, and to test for six mutations accounting for $85 \%$ of CF chromosomes, ${ }^{3}(2)$ to offer the partners of women who test positive for the CF gene a carrier test, and (3) to offer prenatal diagnosis to heterozygous couples.

Many of the arguments about CF carrier screening concern the incompleteness of screening. Because the test fails to account for $15 \%$ of CF mutations, couples in which neither partner has an identifiable mutation have a residual 1 in 104000 risk of having an affected child. However, in approximately $4 \%$ of couples the women will test positive and her partner negative. These couples face a 1 in 640 risk of having a CF child, substantially greater than their starting risk of 1 in 2500 . This study aimed to measure the psychological impact of prenatal CF carrier testing on those couples faced with this intermediate risk.

\section{Subjects and methods}

Women up to 18 weeks' gestation presenting for antenatal care at the Simpson Memorial Maternity Pavilion, Edinburgh, were eligible for inclusion in the screening trial. Details of the recruitment and screening method are given elsewhere. ${ }^{5}$ The protocol and administration of psychological tests is outlined in fig 1.

From May 1991 to January 1992 a total of 1798 women was screened and $69(4 \%)$ were identified as CF carriers. In all cases the male partner was screened. Excluded from this study were three couples where both partners proved heterozygous. Further exclusions were one couple who suffered a pregnancy loss, one couple who failed to complete the questionnaires, and one screened partner who requested exclusion from the study even though his carrier partner expressed a wish to be included. A total of 64 carrier women and 63 male partners entered the study. One couple subsequently separated resulting in only 62 partners completing the final questionnaires.

For each carrier, two control subjects of the same parity were selected. Control subjects had attended the same antenatal booking clinic 


\section{SCREENING PROTOCOL}

Booking clinic appointment

pre-screening information leaflet self-administered pre-screening questionnaire threshold GHO

Threshold stage:

Booking clinic

pre-screening counselling (midwife) mouth wash sample and consent form collect questionnaire

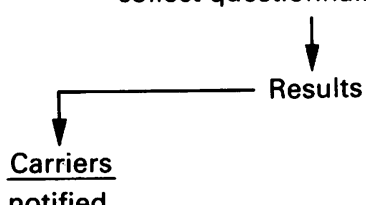

1

Stage 1:

Counselling appointment carrier
partner
GHQ1, SRT1)

mouth wash sample and consent form

Stage 2: Partner's results notified (GHQ2, SRT2)

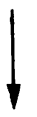

Stage 3:

6 weeks post-test

(GHO3, SRT3)<smiles>[3H][3H]</smiles>

Stage 4:

6 weeks postnatal

(GHO4, SRT4)
Non-carriers notified if requested (GHO1, SRT1)

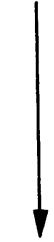

(GHO2, SRT2)

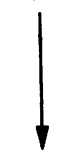

(GHO3, SRT3)

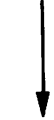

(GHO4, SRT4)

Figure 1 Screening protocol and administration of psychological tests.

as the carrier, had received a negative test result, and had a male partner willing to act as a control subject. A total of 116 female controls and 115 male controls agreed to participate. Of these, 13 couples failed to complete all the questionnaires and two suffered a pregnancy loss. A total of 101 female controls and 100 male controls completed the study to stage 3 . Failure to trace two couples resulted in 99 female controls and 98 male controls completing the final questionnaires.

\section{Measures}

Sociodemographic data were obtained from subjects' antenatal records. A self administered pre-screening questionnaire assessed perceived carrier risk and emotional response to screening. Threshold psychological assessment and the impact of screening on carriers was assessed by two self rating questionnaires, the General Health Questionnaire (GHQ) and the Symptom Rating Test (SRT).

The $\mathrm{GHQ}^{6}$ is a screening tool which identifies two main classes of problem: inability to carry out one's normal 'healthy' functions and the appearance of new phenomena of a distressing nature. Given that time is at a premium in an antenatal booking clinic the

shortest version, the 12 item GHQ, was chosen using the $3 / 4$ cut off point. ${ }^{7}$

The nature of psychological response was assessed by the SRT. ${ }^{8}$ Composed of 30 symptoms briefly defined in simple language, the SRT allows separate scores to be derived for anxiety, depression, inadequacy, and somatic symptoms. Unlike the $\mathrm{GHQ}$, it is not a case finding instrument but will measure psychological distress and is very sensitive to change.

The GHQ scored respondents as positive or negative. The significance of differences between groups was evaluated by the $\chi^{2}$ test. The SRT ascribed scores to subjects. As the scores were not normally distributed (skew to higher values), the significance of differences between groups was assessed by the median test.

\section{Procedure}

An information leaflet was sent to all antenatal patients with their booking clinic appointment. Details of the leaflet have been described previously. ${ }^{9}$ Enclosed with the leaflet was a pre-screening questionnaire incorporating a GHQ (termed threshold GHQ). Thus, women suffering from psychological disturbance before receiving a positive CF test result could be ascertained. Women were invited to complete the questionnaire at home and to bring it with them to the clinic. Those women entering the trial who had not completed the questionnaire at home were asked to complete one at the clinic.

Pre-screening counselling and obtaining a mouthwash sample for DNA analysis was carried out by the midwife responsible for booking the patient. Activity at the clinic limited data collection, so only women presenting with a positive GHQ were asked to complete a SRT (termed threshold SRT) to determine the nature of their distress. These women were interviewed by a genetic nurse to ascertain the likely source of their psychological disturbance. GHQ and SRT scores along with interview data were stored on a computer database for ease of storage and recall when a carrier was ascertained.

Women identified as CF carriers were contacted a week later by telephone or, in a minority of cases, by letter and invited to attend the hospital for counselling along with their partner. The couple were seen by a genetic nurse who, before counselling, asked each partner to complete a GHQ and a SRT (termed GHQ1 and SRT1). Counselling was carried out using visual aids and couples were given a detailed information leaflet with a contact telephone number. A consent form and a mouthwash sample for DNA analysis were obtained from the partner.

On receipt of the partner's negative test result (average four days) the couple were contacted in all cases by telephone and informed of the result. A letter was sent confirming the partner's negative test result and reiterating the residual risk of 1 in 640 of having an affected child. Enclosed were a stamped addressed envelope and a GHQ and a 
Table 1 Sociodemographic data (percentages in brackets).

\begin{tabular}{lrrr}
\hline & $\begin{array}{c}\text { Total } \\
\text { population } \\
(\mathbf{n}=1798)\end{array}$ & $\begin{array}{c}\text { Carriers } \\
(\mathbf{n}=64)\end{array}$ & $\begin{array}{c}\text { Selected } \\
\text { controls } \\
(\mathbf{n}=101)\end{array}$ \\
\hline Age (y) & $28 \cdot 07$ & $27 \cdot 86$ & $28 \cdot 64$ \\
Mean & $16-44$ & $18-44$ & $20-40$ \\
Range & & & \\
Parity & $916(51)$ & $35(55)$ & $52(51)$ \\
Primiparous & $882(49)$ & $29(45)$ & $49(49)$ \\
Multiparous & & & \\
Gestation (wk) & $12 \cdot 25$ & $11 \cdot 94$ & $12 \cdot 25$ \\
Mean & $6-18$ & $7-16$ & $7-18$ \\
Range & & & \\
Marital status & $1316(73)$ & $48(74)$ & $82(81)$ \\
Married & $409(23)$ & $14(22)$ & $16(18)$ \\
Single & $46(3)$ & $0(0)$ & $3(3)$ \\
Divorced & $24(1)$ & $1(2)$ & $0(0)$ \\
Separated & $3(-)$ & $1(2)$ & $0(0)$ \\
Widowed & & & \\
Social class & & & \\
1 & $227(13)$ & $5(8)$ & $11(11)$ \\
2 & $553(31)$ & $18(28)$ & $31(31)$ \\
3 & $601(33)$ & $23(36)$ & $36(35)$ \\
4 & $158(9)$ & $10(16)$ & $16(16)$ \\
5 & $91(5)$ & $4(6)$ & $3(3)$ \\
Unemployed & $141(8)$ & $4(6)$ & $3(3)$ \\
Student & $27(1)$ & $0(0)$ & $1(1)$ \\
\hline & & &
\end{tabular}

SRT (termed GHQ2 and SRT2). Six weeks later the couple were sent a further postal GHQ and a SRT (termed GHQ3 and SRT3) and finally six weeks after delivery the same two measures were sent (termed GHQ4 and SRT4).

Control couples received a postal GHQ and a SRT at comparable intervals to carriers and partners.

Table 2 Perception of carrier risk and emotional response to screening (percentages in brackets).

\begin{tabular}{lrcrr}
\hline \multirow{2}{*}{$\begin{array}{l}\text { Perception } \\
\text { of risk }\end{array}$} & \multicolumn{4}{c}{ Emotional response* } \\
\cline { 2 - 5 } & Anxious & Reassured & Don't know & \multicolumn{1}{c}{ Total } \\
\hline 1 in 4 & $78(24)$ & $154(47)$ & $97(29)$ & $329(100)$ \\
1 in 25 & $258(24)$ & $533(51)$ & $264(25)$ & $1055(100)$ \\
1 in 100 & $7(35)$ & $13(65)$ & 0 & $20(100)$ \\
1 in 200 & $1(6)$ & $11(69)$ & $4(25)$ & $16(100)$ \\
Don't know & $78(21)$ & $169(45)$ & $129(34)$ & $376(100)$ \\
\hline
\end{tabular}

* Two subjects no response.

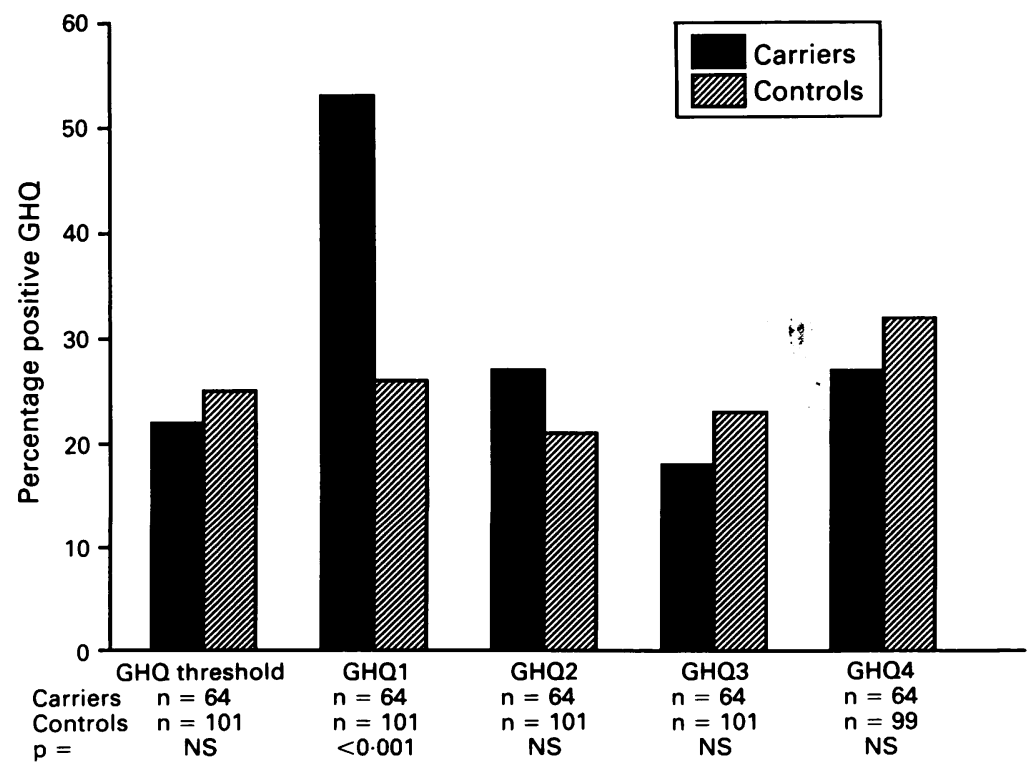

Figure $2 G H Q$ results. Percentages of carriers and controls with positive responses $(3+)$ shown for each assessment point. Numbers of carriers and controls tested shown below figure.

\section{Results}

SOCIODEMOGRAPHIC DATA

The sociodemographic characteristics of the screened population, carriers, and controls are shown in table 1 . The sample was weighted toward the higher socioeconomic groups (assessed from the occupation of the head of the household using the Registrar General's classification). This reflects the tendency for women of the higher social classes to present earlier for antenatal care and to have a stable partnership. Furthermore, a majority of women from a sizeable area housing those of the lower socioeconomic groups booked for antenatal care at a clinic within that area.

\section{ANXIETY AND PERCEIVED CARRIER RISK}

The pre-screening questionnaire assessed perceived carrier risk. Five carrier risk options were stated and women were asked to select one (table 2). Of the 1798 women screened, $1055(59 \%)$ perceived their risk correctly. A substantial number $(378,21 \%)$ had no perception of their risk, and a minority $(36,2 \%)$ perceived their risk to be considerably lower than the 1 in 25 risk stated in the pre-screening information leaflet. A further 329 (18\%) perceived their risk to be 1 in 4 .

Asked to indicate their emotional response to being screened, $880(49 \%)$ stated they were reassured and $494(28 \%)$ did not know how they felt. Anxiety was felt by $422(23 \%)$ of women and there was no response in two cases. There was no correlation between anxiety about being screened and perceiving one's risk to be higher than 1 in 25 (table 2).

\section{RESULTS ON THE GENERAL HEALTH} QUESTIONNAIRE (GHQ)

Preliminary results of GHQ data on carrier and control subjects have been reported elsewhere..$^{5}$ In this extended study a total of 576 $(32 \%)$ of the screened population presented with a positive threshold GHQ. Many women $(44 \%)$ cited symptoms of pregnancy as the problem. Twelve percent stated their pregnancy was unplanned, $10 \%$ had a poor obstetric history, and a further $7 \%$ felt generally anxious about the pregnancy. Two percent were worried about other antenatal diagnostic tests but only two women were concerned about the CF screening test. Four percent of women had a psychiatric history.

Of the 64 carriers, $14(22 \%)$ had a positive GHQ against $25(25 \%)$ among the controls (fig 2 ). On receiving positive test results, the proportion of carriers $(53 \%)$ with a positive GHQ1 score was significantly greater than the proportion of control subjects $(27 \%)\left(\chi^{2}\right.$, $\mathrm{p}<0.001)$. At the time of their partner's negative test result (GHQ2), at six weeks after the test (GHQ3) and at six weeks after delivery (GHQ4), carriers showed no significant difference in the proportion of positive scores when compared to selected controls (fig 2).

No significant difference was found between the proportion of partners and their selected controls with a positive GHQ score at any of 
the four assessment points (fig 3). However, 14 of $15(93 \%)$ partners with a positive GHQ1 had a female partner who had also scored positive. Nine of the $10(90 \%)$ male controls who had positive GHQ1 scores had a female partner with a positive GHQ score. Males were, therefore, significantly more likely to present with psychological distress if their female counterpart was also distressed $\left(\chi^{2}\right.$, $\mathrm{p}<0.001)$.

\section{SYMPTOM RATING TEST (SRT)}

Five hundred and nineteen of the $576(90 \%)$ of the total screened population with a positive threshold GHQ were interviewed and completed a threshold SRT. Among this group were $14(22 \%)$ carriers and $25(25 \%)$ selected controls. There was no significant difference in the threshold SRT scores of the three groups. However, at SRT1 (carrier receiving positive test result) there was a significant difference between carriers and controls in the total score for generalised psychological disturbance (median test, $\mathrm{p}<0.005$ ) and specifically in the subscores for anxiety and depression (median test, $\mathrm{p}<0.001$, table 3 ).

On receiving their partner's negative test result (SRT2) the scores of carriers returned to control levels and remained there at the six week post-test point (SRT3) and again at the six week post-delivery point (SRT4) (table 3).

There was no significant difference between SRT scores for generalised psychological disturbance of the partners of carriers when compared with their selected controls (table 4). Anxiety and inadequacy subscores were significantly higher than those of controls at the time when carriers were given their positive test results (median test, $\mathrm{p}<0.05$ and $\mathrm{p}<0.02$ respectively, table 4 ).

There was a significant decrease in the subscores of anxiety in SRT1 and SRT2 scores in

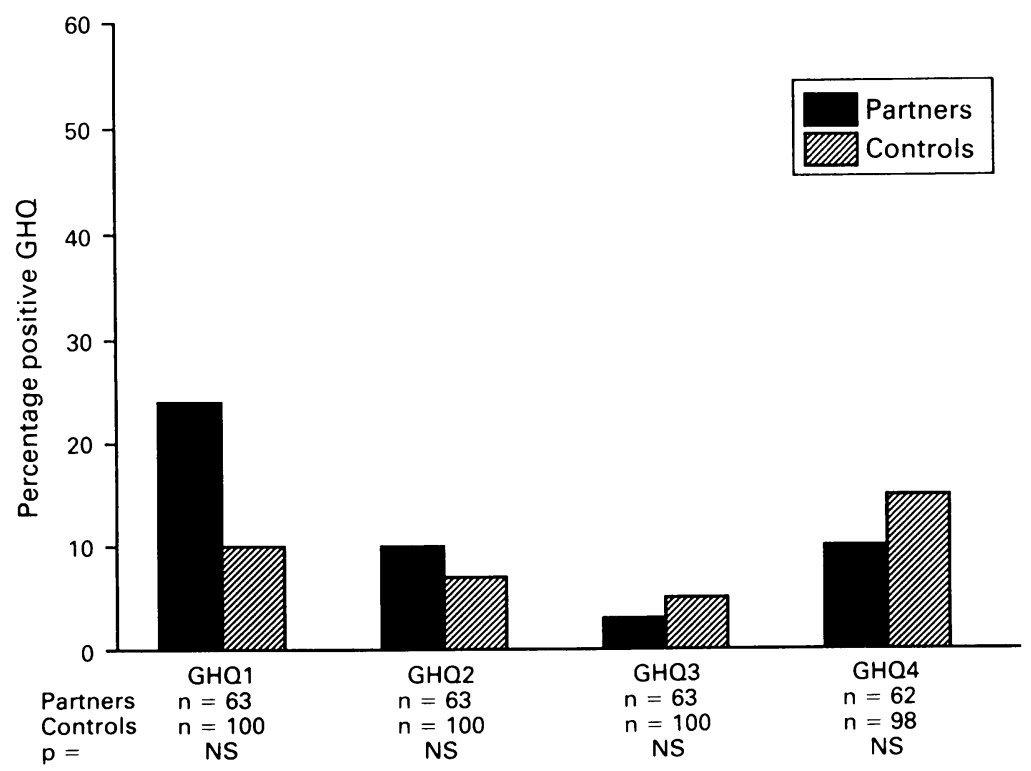

Figure $3 G H Q$ results. Percentages of partners and controls with positive responses $(3+)$ shown for each assessment point. Numbers of partners and controls tested shown below figure.
Table 3 Median of symptom rating test ( $S R T$ ) scores of carriers and controls.

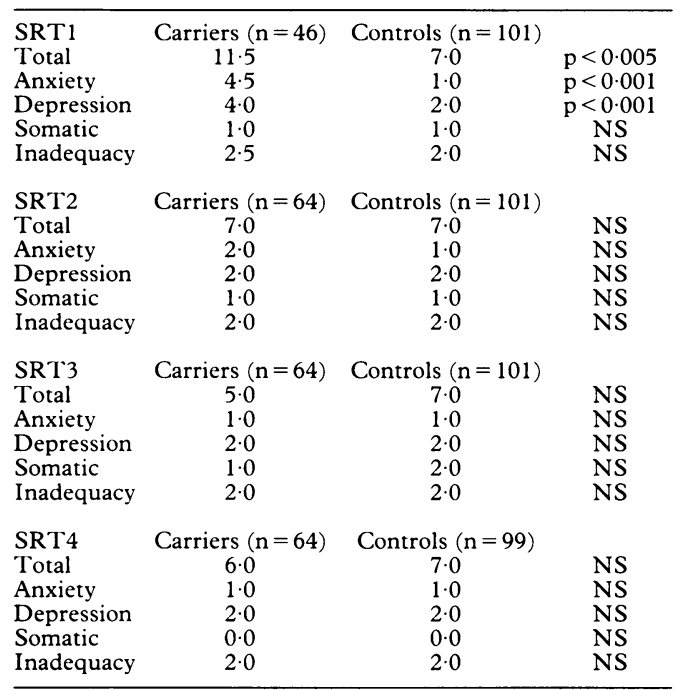

Table 4 Median of symptom rating test (SRT) scores of partners and controls.

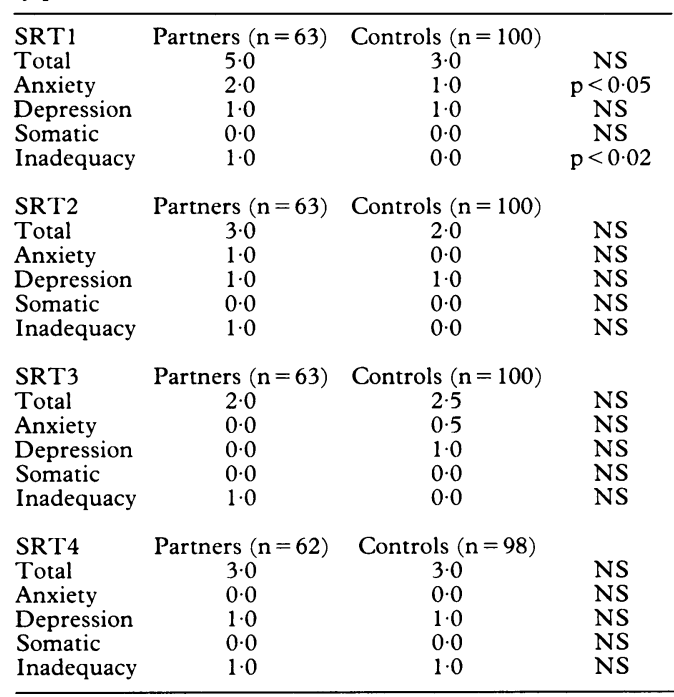

both carriers and their partners (table 5). This also showed up in the subscores for depression among carriers.

\section{Discussion}

The findings from this study show that prenatal carrier screening delivered in two stages does generate some psychological disturbance. In the identified carrier there is a significant

Table 5 Comparison of median of SRT1 and SRT2 scores in carriers $(n=64)$ and partners $(n=63)$.

\begin{tabular}{lccc}
\hline Carrier's scores & SRT1 & SRT2 & \\
Total & 11.5 & 7.0 & $\mathrm{NS}$ \\
Anxiety & 4.5 & 2.0 & $\mathrm{p}<0.001$ \\
Depression & 4.0 & 2.0 & $\mathrm{p}<0.02$ \\
Somatic & 1.0 & 1.0 & $\mathrm{NS}$ \\
Inadequacy & 2.5 & 2.0 & $\mathrm{NS}$ \\
& & & \\
Partner's scores & SRT1 & SRT2 & \\
Total & 5.0 & 3.0 & $\mathrm{NS}$ \\
Anxiety & 2.0 & 1.0 & $\mathrm{p}<0.005$ \\
Depression & 1.0 & 1.0 & $\mathrm{NS}$ \\
Somatic & 0.0 & 0.0 & $\mathrm{NS}$ \\
Inadequacy & 1.0 & 1.0 & $\mathrm{NS}$ \\
\hline
\end{tabular}


increase in generalised psychological disturbance, specifically anxiety and depression, when compared to a control population. This reaction occurs in response to learning of their carrier status and lasts for the period (approximately four days in this study) awaiting their partner's test result. On receiving their partner's negative test result the distress subsides and there is no indication of a resurgence during the pregnancy or in the immediate postnatal period. Although the longer term effects are unknown, three carriers from this study have subsequently embarked upon a further pregnancy.

Male partners manifested symptoms of anxiety and inadequacy during the period awaiting their test result, but this disappeared on receipt of a negative result. Both partners and male control subjects were significantly more likely to manifest psychological disturbance if their female partner was distressed.

Thirty-two percent of women presented at the antenatal booking clinic with a positive GHQ score. This is comparable with other studies. ${ }^{10}$ Previous work has shown that randomly selected samples from the community will contain quite high proportions of persons with degrees of psychological disturbance ranging from mild to severe. ${ }^{11}$ Indeed it is estimated that $25 \%$ of patients seen in general practice have anxiety as a clinically significant component of their condition. ${ }^{12}$

In this study subjects presenting with negative GHQ scores submitted mean SRT scores comparable with normal subjects in previous studies ${ }^{813}$ Carriers with positive GHQ1 scores submitted SRT1 scores well below those reported for psychiatric patients ${ }^{814}$ and comparable with scores generated by the Symptom Questionnaire in patients undergoing amniocentesis. ${ }^{14}$ This is a point worth noting as previous studies have suggested that levels of anxiety in pregnant women who receive a positive test result can be extremely high, above those for psychiatric patients. ${ }^{15}$ It is clear that a substantial number of women will enter a prenatal screening programme with concurrent stress. Indeed, five of the $14 \mathrm{car}$ riers who presented with a positive threshold GHQ score maintained these scores throughout; for reasons of recent bereavement (three cases), diagnosis of chronic illness in the partner (one case), and regular ECG monitoring throughout pregnancy for attacks of breathlessness (one case). Eleven out of 25 control subjects who presented with positive threshold scores maintained these scores throughout the study for a variety of reasons. Threshold psychological assessment on all women screened served not only to ensure for the purposes of this study that there was no significant psychological difference between carriers and control subjects at the outset, but proved valuable in the wider screening trial for ascertaining women identified as CF carriers who were already experiencing stress and might require extra counselling and support.

Previous studies on patients undergoing prenatal screening have indicated that once a woman perceives her pregnancy has been threatened she continues to be concerned. ${ }^{16}$ Conversely, others have shown a dramatic return to normal once a negative test result is given. ${ }^{141718}$ The results of this study agree with the findings of the latter. A notable effect of prenatal CF carrier screening upon two carriers was their subsequently declining $\alpha$ fetoprotein (AFP) screening. A further two carriers received abnormal AFP screening results and underwent amniocentesis for chromosome studies. Despite this only one of these four women was included in the $6 \%$ of carriers who stated they were against the test being offered routinely to pregnant women. A concern must be that some women may not be so resilient to multiple provoking agents during pregnancy, which could foreseeably occur if a woman experiences several positive screening test results, and particularly were she already suffering from concurrent stress. A positive aspect is that the developing area of prenatal screening will help us to consider more carefully the whole area of psychosocial support in the provision of antenatal care, which some perceive to have been neglected. ${ }^{19}$

Psychological disturbance is considered a normal rather than a pathological response to prenatal diagnosis. ${ }^{20}$ It has been suggested that counselling efforts should support the person's attempt to cope with stress accompanying the procedure rather than not provide screening. ${ }^{21}$ The results of this study suggests that stress resulting from being identified as a CF carrier during pregnancy is short lived and that those couples faced with an intermediate risk of having a CF child cope satisfactorily.

We thank the antenatal patients who responded to this study and the medical and midwifery staff of the Simpson Memorial Maternity Pavilion for making this trial possible. The study was funded by grants from The Cystic Fibrosis Trust and the Scottish Home and Health Department.

1 Kerem BS, Rommens JM, Buchanan JA, et al. Identification of the cystic fibrosis gene: genetic analysis. Science 1989;245:1073-80.

2 Watson EK, Mayall ES, Simova L, et al. The incidence of delta F508 mutation, and associated haplotypes, in a sample of English CF families. Hum Genet 1990;85:4356.

3 Shrimpton AE, McIntosh I, Brock DJH. The incidence of different cystic fibrosis mutations in the Scottish population: effects on prenatal diagnosis and genetic counselling f Med Genet 1991;28:317-21.

4 Watson EK, Mayell E, Chapple J, et al. Screening for carriers of cystic fibrosis through primary health care services. BMF 1991;303:504-7.

5 Mennie ME, Gilfillan A, Compton M, et al. Prenata screening for cystic fibrosis. Lancet 1992;340:214-16.

6 Goldberg D, Williams P. A user's guide to the General Health Questionnaire. Windsor: NFER-Nelson, 1988.

7 Mari J, Williams P. A comparison of the validity of two psychiatric screening questionnaires (GHQ12 and SRQ20) in Brazil, using relative operating characteristic SRQ20) in Brazil, using relative operat
analysis. Psychol Med 1985;15:651-9.

8 Kellner R, Sheffield BF. A self-rating scale of distress. Psychol Med 1973;3:88-100.

9 Mennie M, Liston WA, Brock DJH. Prenatal cystic fibrosis carrier testing: designing an information leaflet to mee the specific needs of the target population. $\mathrm{F}$ Med Gene 1992;29:308-12.

10 Sharp D. Validation of the 30-item General Health Questionnaire in early pregnancy. Psychol Med 1988;18:503-7. 11 Goldberg D, Huxley P. Common mental disorders. A biosocial model. Tavistock: Routledge, 1992.

12 Wilkinson $\mathrm{G}$. Anxiety: recognition and treatment in general practice. Oxford: Radcliffe Medical Press Ltd, 1992

13 Cochrane R. A comparative evaluation of the Symptom Rating Test and the Langer 22-item Index for use in epidemiological surveys. Psychol Med 1980;10:115-24. 14 Fava G, Kellner R, Perini G, et al. Italian validation of the 
Symptom Rating Test (SRT) and Symptom Questionnaire. Can f Psychiatry 1983;28:117-23.

15 Robinson JO, Hibbard BM, Laurence KM. Anxiety during a crisis: emotional effects of screening for neural tube defects. F Psychosom Res 1984;28:163-9.

16 Tabor A, Jonsson MH. Psychological impact of amniocentesis in low risk women. Prenat Diagn 1987;7:443-9.

17 Burton BK, Dillard RG, Clark EN. The psychological impact of false positive elevations of maternal serum alpha-fetoprotein. Am $\mathcal{F}$ Obstet Gynecol 1985;151:77-82.

18 Tsoi $M M$, Hunter $M$, Pearce $M$. Ultrasound scanning in women with raised serum alpha-fetoprotein: short term women with raised serum alpha-fetoprotein: short tern
psychological effects. $\mathcal{f}$ Psychosom Res 1981;31:35-9.
19 Campbell IE, Field PA. Common psychological concerns experienced by parents during pregnancy. Can Ment Health 1989;37:2-5.

20 Blumberg B. The emotional implications of prenatal diagnosis. In: Emery AEH, Pullen I, eds. Psychological aspects of genetic counselling. London: Academic Press 1988: 202-7.

21 Childs B, Gordis L, Kaback M, Kazazian HH. Tay-Sachs screening: social and psychological impact. Am f Hum Genet 1976;28:550-8. 